\title{
ReMed: rapport annuel 2017
}

ReMed, Comité de direction

\section{ReMed - le réseau de soutien pour les médecins}

Si les médecins œuvrent au maintien et au rétablissement de la santé de leurs patients, leur propre santé devrait toutefois également être prise en compte car la médecine est une profession exigeante; les médecins peuvent être soumis à une pression telle qu'elle affecte parfois leur santé physique et psychique. ReMed est un réseau de soutien destiné aux médecins qui leur transmet ses connaissances et ses expériences en faveur de la promotion de la santé et de la prévention ceci afin de les sensibiliser à leur propre santé. ReMed dispose d'un large éventail de prestations pour épauler les médecins en situation de crise. Son objectif est de maintenir les médecins en bonne santé, de préserver leur capacité fonctionnelle afin de garantir la sécurité des patients et une qualité élevée des soins à la population.

Contexte: ReMed est une organisation autonome du corps médical financée par la Fédération des médecins suisses (FMH). Le réseau ReMed base ses procédures sur les prescriptions légales en vigueur et sur le Code de déontologie de la FMH.

\section{ReMed - 141 demandes de prestations en 2017}

La nécessité d'un réseau de soutien comme ReMed pour les médecins en situation de crise a été à nouveau confirmée pendant l'année écoulée. Par rapport à l'année précédente, les demandes ont augmenté de $25 \%$ (soit 141 demandes). Les médecins du Comité de direction ont répondu à 107 demandes de Suisse alémanique et 34 de Suisse romande parmi lesquelles $61 \%$ concernaient des femmes et 39\% des hommes répartis environ pour moitié entre le domaine ambulatoire et hospitalier. Dans ce dernier domaine la majorité sont des jeunes médecins en formation postgraduée ce que nous trouvons particulièrement préoccupant pour le futur.

\section{ReMed - retour sur l'offre de conseil}

Les médecins qui se sont adressés à ReMed ont été nombreux à recourir à la possibilité de donner un avis anonyme sur la qualité du premier entretien. Ces retours permettent aux premiers répondants d'amélio- rer la qualité du conseil et contribuent à développer le programme.

\section{ReMed - évaluation achevée}

Après huit ans d'existence, ReMed a fait l'objet d'une évaluation par une institution externe dans le but de disposer de bases concrètes pour décider de la forme à donner au ReMed de demain. Dans ce contexte, plus de $90 \%$ des médecins interrogés ont estimé que l'offre ReMed correspondait à un besoin du corps médical. Parmi les médecins ayant fait appel à ReMed, près de 90\% d'entre eux se sont déclarés satisfaits du temps de réponse suite à la première prise de contact. L'évaluation a également permis de montrer que ReMed atteint ses objectifs en matière d'intervention de crise et de prévention.

\section{Programme ReMed - adéquation}

Les offres, les besoins et l'organisation de ReMed sont coordonnés. ReMed a à cœur d'améliorer et d'élargir continuellement son offre. En 2017, le réseau a ainsi été renforcé et élargi. Les premiers répondants, les membres du réseau et les experts répondants se sont rencontrés à plusieurs reprises durant l'année écoulée pour présenter des cas et échanger leurs expériences. En raison de leur succès, des rencontres supplémentaires seront proposées pour les premiers répondants en 2018.

\section{ReMed - groupes de parole}

Le projet-pilote des groupes de coaching lancé en 2016 a été reconduit avec succès en 2017. Les groupes de parole à Berne et Zurich animés par un expert ont permis d'offrir, dans une ambiance collégiale, un soutien aux médecins qui doivent faire face aux défis et sollicitations liés à leur activité professionnelle. L'offre s'adresse aux médecins confrontés à des sujets difficiles et délicats ou à de gros défis, dans but de leur permettre d'exprimer les conflits et difficultés propres à leur travail et trouver des solutions appropriées. En 2018, ces rencontres seront proposées à Berne, Zurich et Saint-Gall et prochainement aussi en Suisse romande. 


\section{ReMed - assurer notre visibilité}

ReMed a continué à accroître sa notoriété en participant à de nombreux congrès en Suisse et à l'étranger. Le réseau de soutien a fait connaître son offre au travers d'ateliers et de représentations théâtrales contribuant ainsi fortement à lever le tabou sur les sollicitations professionnelles auxquelles les médecins sont confrontés. En 2017, ReMed a également assuré sa présence dans les médias par la publication d'articles. Les témoignages publiés dans le Bulletin des médecins suisses rencontrent un grand succès et un écho positif auprès des lecteurs et montrent comment ReMed est venu en aide aux personnes concernées.

\section{ReMed - site Internet remanié}

L'année dernière, le site de ReMed a été remanié et mis à jour. Une vidéo explicative accueille désormais les visiteurs leur présentant les motifs d'une prise de contact.

Sur le site www.swiss-remed.ch, vous découvrirez des rapports d'expérience passionnants, de la documentation et des informations précieuses concernant le réseau de soutien.

Le programme national ReMed avec le Comité de direction et le réseau d'intervenants sera aussi là en 2018 pour les médecins en situation de crise. Les médecins qui le souhaitent peuvent prendre contact $24 \mathrm{~h}$ sur 24 via la ligne d'assistance (0800 0 73633) ou par courriel à help@swiss-remed.ch
ReMed

Secrétariat

CH-3000 Berne

Tél. 0313591200

info[at]swiss-remed.ch

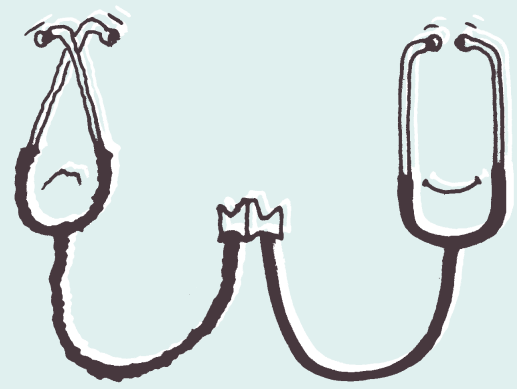

Et soudain, vous aussi! Les crises touchent aussi les médecins.

Acceptez de l'aide. Contactez ReMed.

Assistance téléphonique $24 \mathrm{~h}$ sur 24

0800073633 help@swiss-remed.ch

08000 ReMed www.swiss-remed.ch 